\title{
The Tension Control System Research of Printing Machine Based On the Movement Theory
}

\author{
Shengjiang Chen ${ }^{1}$, Chunfeng Zhang ${ }^{2, *}$ \\ ${ }^{1}$ School of Nuclear Technology and Chemistry \& Biology, Hubei University of Science and Technology, \\ Xianning, Hubei, China \\ ${ }^{2}$ School of Foreign Languages, Hubei University of Science and Technology, Xianning, Hubei, China \\ *Corresponding author
}

Keywords: movement theory, printing machine, tension, control system

\begin{abstract}
Based on the theory of movement printing machine tension control way and principle is analyzed, and combining with the actual production of equipment failure, according to the principle of tension system fault screening and maintenance so as to solve practical problems. On the basis of movement s theory, part of the implementation of the system, this paper puts forward the ideas of modification using torque motor to replace, do optimize structure, and improve the control precision of purpose.
\end{abstract}

\section{Introduction}

Printing and packaging industries in our country in recent years has achieved rapid development, face unprecedented huge opportunities, and also faces great challenge. The continuous improvement of printing speed for printing a tension control system is becoming more and more demanding [1]. The use of magnetic powder clutch to transfer torque of the traditional control mode limits the speed of operation, also the waste of resources, for the clutch, torque is large (large) of coil diameter sliding speed is bigger, easy to produce the adverse situation, such as fever and the reasons of its own life, caused the high failure rate, operate at a high speed printing machine, also caused a big waste of paper.

\section{Printing machine tension control system principle and analysis}

Summary of the tension. Printing press is a kind of special machinery and equipment, it will printing liquid evenly on the movement of the support body, make its have some new USES or reach some special performance, and enables the coating thickness and uniformity of coating products, coating properties such as adhesive fastness and support body and the speed of printing products to meet the design requirements [1].

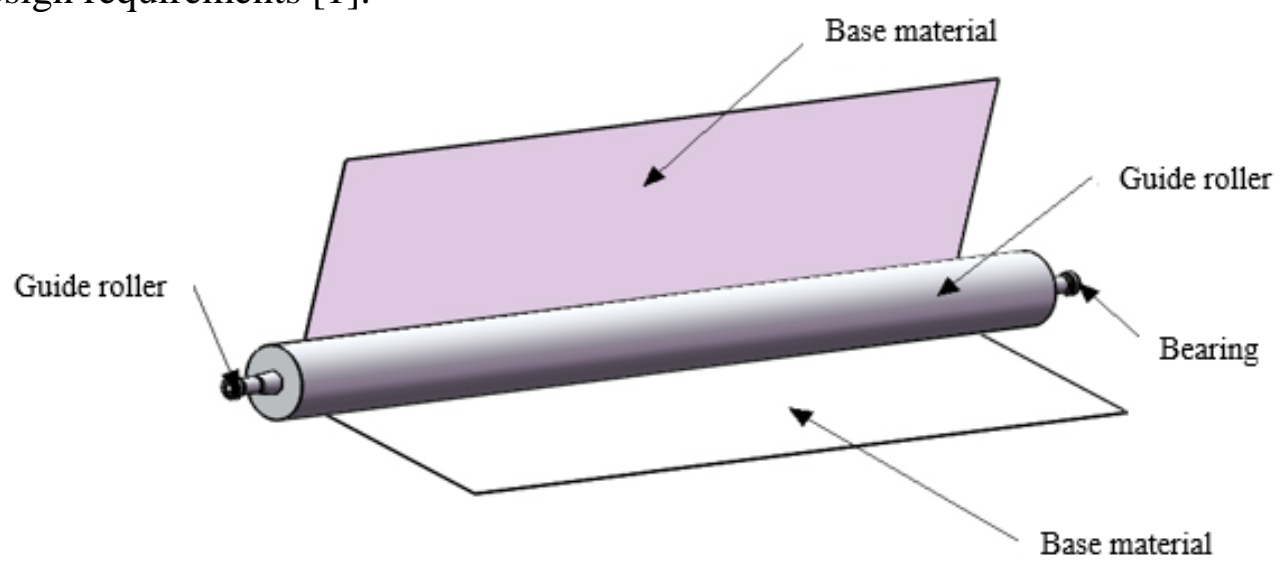

Fig. 1 The main structure of printer 
Printing machine is the main structure by supporting body or base material on multiple guide roller winding form a certain way, and will produce certain tension substrate tension of equipment to run. As shown in Fig. 1 below.

Printing equipment mainly refers to the printing press and its corresponding auxiliary system, includes printing presses, drying system, three parts for winding and auxiliary system.

Printer: the print head is its important part, its different structure or form determines the different ways of printing.

Drying device: printing and drying process is the printing project has continued for two process. It also formed two most printing equipment, printing speed fast, often limited by drying capacity, and the size of the drying ability and top speed as the design basis to the printer. By air drying, the current main, using treated dry air as a medium for dries form.

Auxiliary equipment: mainly including feeder system, drive system, tension control device, electrostatic removing device, adjusting device, etc.

Make the base material movement in the printing press is the source of the tension device and tension control system, it is printing equipment can normal production and production of qualified products one of the main factors. The study of tension system more in-depth, more can ensure good use of the equipment.

\section{Printing machine tension control system}

This system is through the feedback signal of tension detector system to determine whether a tension reached set value, and through the coordination of tension roll before and after the synchronicity of the running speed of the servo motor to ensure the tension stability.

Control system of the detectors contains a tension. There is a kind of system besides containing tension tester, also including floating roller, pressurization valve and cylinder device [2]. This system can be used as shown in figure 2 graphics to represent:
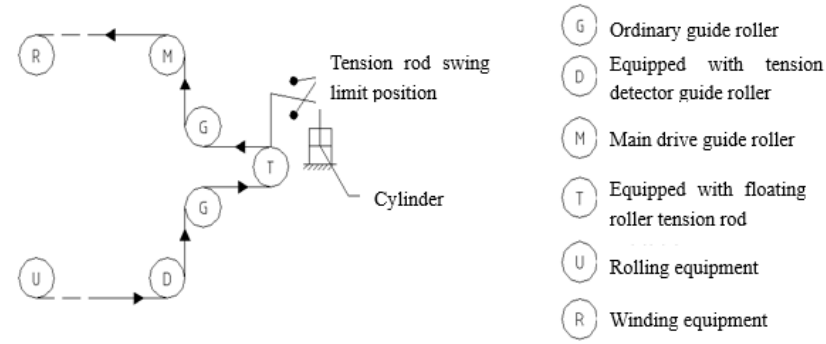

Fig. 2 Contains tension and roller detector control system diagram

This system through the feedback signal of tension detector system to determine whether a tension reached set value, and through the small output voltage or current signal amplifier amplification process to control a pressurization valve, thus filling the corresponding compressed air to the cylinder loading of tension to balance the tension on the roll, achieve the purpose of tension control system, at the same time, ensure the tension stability [3]. The mechanical model is shown in figure 3 below, and its dynamic equation based on this model can be listed [2].

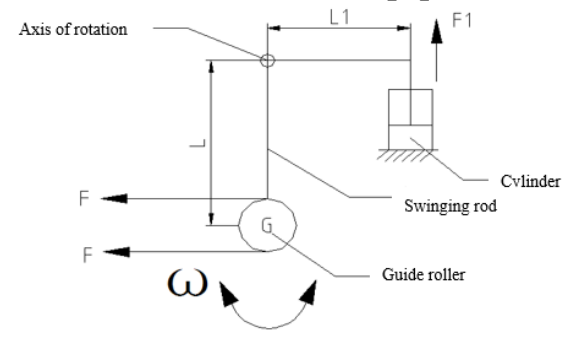

Fig. 3 The mechanical model of tension control

Support system tension for F, cylinder is F1, the rotational inertia of the guide roller and swinging rod tension, guide roller and swinging rod rotation angular velocity is $\omega$, guide roller is the quality of 
the $\mathrm{m} 1$, swinging rod quality is $\mathrm{m} 2$, in order to guide roller and swinging rod as the research object, can obtain the dynamic equation,

$$
\begin{array}{r}
2 \mathrm{FL}-\mathrm{F}_{1} \mathrm{~L}_{1}=I \frac{d \omega}{d t} \\
\rightarrow \mathrm{F}=\frac{\mathrm{F} 1}{2} \cdot \frac{\mathrm{L} 1}{\mathrm{~L}}+\frac{I}{2 L} \bullet \frac{d \omega}{d t}
\end{array}
$$

Since the moment of inertia is,

$$
I=\mathrm{m} 1 \mathrm{~L} 2+\frac{1}{3} \mathrm{~m} 2 \mathrm{~L} 2
$$

Then, combine formula (2) and (3),

$$
\begin{array}{r}
\mathrm{F}=\frac{\mathrm{F} 1}{2} \cdot \frac{\mathrm{L} 1}{\mathrm{~L}}+\frac{1}{6}(3 \mathrm{~m} 1+\mathrm{m} 2) \bullet \mathrm{L} \bullet \frac{d \omega}{d t} \\
\mathrm{~F}=\frac{\mathrm{F} 1}{2} \cdot \frac{\mathrm{L} 1}{\mathrm{~L}}+\frac{1}{6}(3 \mathrm{~m} 1+\mathrm{m} 2) \cdot \frac{d \omega L}{d t}
\end{array}
$$

As Linear velocity $v=\omega \mathrm{L}$,

So formula (4) can be transform as

$$
\mathrm{F}=\frac{\mathrm{F} 1}{2} \cdot \frac{\mathrm{L} 1}{\mathrm{~L}}+\frac{1}{6}(3 \mathrm{~m} 1+\mathrm{m} 2) \cdot \frac{d v}{d t}
$$

By the above analysis of the dynamic equation can know system tension $\mathrm{F}$ main cylinder support F1 and linear velocity $v$ is directly related to the rate of change of $\frac{d v}{d t}$. That is to say, if under the frontal drag system of equipment, base material not run-time speed constant is a variable speed, cylinder and output of support must be adjusted in time, so as to ensure the stability of the system tension F.

\section{Tension control of printing the important meaning and function}

Printing machine tension control is the core of the whole machine. Small, as long as stable tension control, tension of the printing press alignment precision and rejection rate is easy to control. Tension fluctuation and change had a great influence on the alignment precision; particularly equipment of the higher printing speed, tension control becomes more important [4]. Therefore, if you want to make sure the printer print quality, efficiency and reliability, must be equipped with functional tension control system.

In the process of printing press tension fluctuate and change is more complex, if not timely and accurate under effective control, the comprehensive influence factors will form the tension of the machine parts in an irregular change model. Now its main influencing factors illustrate analysis is as follows:

1) Material in rolling process, and put the diameter of the coil winding and is constantly changing, the diameter of the changes will inevitably cause the change of strip tension. Put the volume under the condition of the brake torque is constant, diameter decreases, and the tension will increase; and winding, on the other hand, if the winding at constant torque as the diameter increases, the tension will decrease. And material is heavier, the faster the speed, the change of strip tension [3]. This is the intrinsic characteristics of the printer. It is one of the main factors of the change of the strip tension.

2) Press the manufacturing precision of the main components and assembly accuracy out-of-tolerance, is also the important factor of the tension caused by fluctuations. Such as base assembly flatness and straightness, wallboard and verticality of base and expressing ideas the 
levelness of the roller and parallelism between each other, and so on. Expressing ideas roller guide roller with many strict parallel with each other, not only in its rotation when they beat quantity deviation and deviation should also ensure quality dynamic and static balance within the scope of the specified value [4]. Otherwise their each roll a week, is expected to take tension will ensue tiny changes accordingly, finally comprehensive reflection on the machine, will lead to the tension produce irregular change. In addition, the printer's main drive system should be no gap, precision transmission, to ensure that each printing unit synchronization operation.

3) Printing machine in the process of non-stop automatically cutting process, material and cutting can make the machine already stable tension produce the interference of sudden change. Machine running speed, the higher the disturbance [5]. Equipment configuration of the tension control system should be able to quickly automatically according to the strip tension perturbation stochastic adjustment, restoring the tension in a timely manner to the original steady state.

4) Strip the in homogeneity of inner material (such as fluctuations in elastic modulus, thickness along the length, the width direction, the eccentric material quality, etc.) as well as the production environment temperature and humidity changes, will be a subtle influence on the whole machine tension fluctuation [5].

\section{Tension system improvement based on the theory of the movement}

In the system due to the presence of pressurization valve and pneumatic components such as air cylinder, has caused a certain degree of instability, shown as in figure 4. If this part of the execution system instead of using torque motor, its installation is simple in form, the reliability of the execution system will have improved greatly [7]. We can use the following diagram said after the transformation of the system.
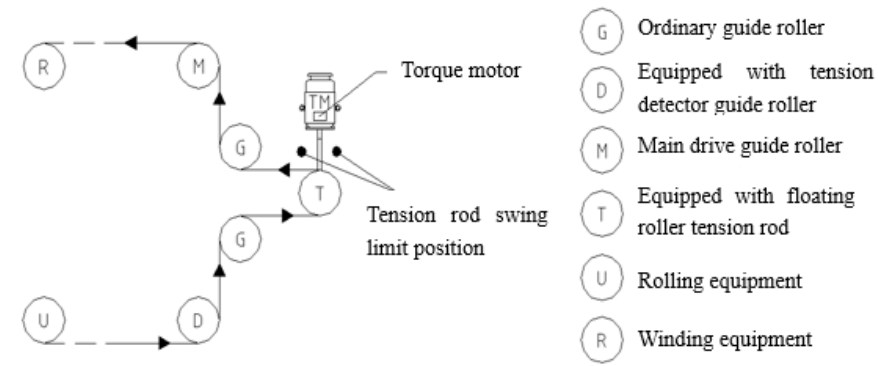

Fig. 4 The improved printing machine tension control system

Through the rotation of the torque motor output torque, to balance the tension of connection is from the floating roller swinging rod on the rotary torque. In practical application are frequent positive and negative to the moment, also can be used to balance positive to torque of the motor output [7]. Of course, from the motor output shaft to the floating roller between need to design some institutions to connect. And this can be installed on machine tail shaft Angle encoder, the motion of the tension rod can be used to detect the Angle value.

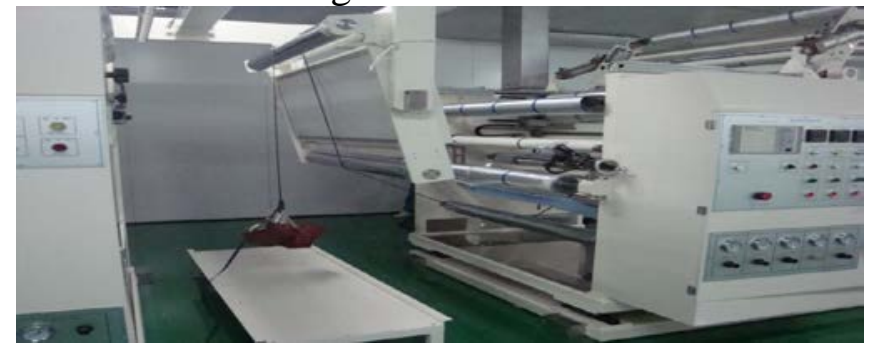

Fig. 5 The improved printing machine tension control system

\section{Summary}

Tension system is a key link in the process of printing equipment and key parts. This paper is to 
research for this system is based on the theory of motion of the printer, the tension system configuration and technique is the mainstream of this field, it should be said. But even so, in the process of practical production also will be more difficult to deal with failure, which requires technical personnel familiar with the structure and working principle of the system, carefully observe the failure phenomenon, analyzing the causes of failure, in the correct way to calibration or maintenance, to ensure that the tension system throughout the printing equipment in the system stable running, not affect the quality of the finished product. Also, in some point of the system chooses higher precision, better stability, upgrade or way more reasonable components, will make the equipment has more development potential.

\section{References}

[1] X. Y. Deng, Tension control in paper machine drives control system of research, Microcomputer information, 2005, vol. 4, pp. 21-24. .

[2] Q. Feng, Tension control technology of printing machine, the management and technology of small and medium-sized enterprise, 2009, vol. 7, pp. 15-18.

[3] Y.B. Zhang, Y.X. Dai and L.T. Ye, Multi-line cutting machine tension control system design, Beijing: journal of mechanical engineering, 2009, vol. 5, pp. 22-24.

[4] Y. Q. Sun, Web press tension control research, Packaging engineering, 2008, vol. 3, pp. 43-48.

[5] R.G. Feng, Modern development and application of printing, Printing technology, 2000, vol. 1, pp. 54-58.

[6] X. Y. Li, Tension control system analysis, programmable controller and factory automation, 2009, vol. 1, pp. 62-65.

[7] Q.Y. Wang, Inference control and its application in automatic windlass tension control, Electrical automation, 2008, vol. 1, pp. 33-36. 